\title{
Non-consistence Aggregation-Disaggregation Technology for Battle Simulation Study of SoS
}

\author{
Yan Xuefei ${ }^{1+}$, Li Qiang ${ }^{1}$, Wang Xiaolong ${ }^{2}$, Liu Dong ${ }^{1}$ and Wang Shoubiao ${ }^{1}$ \\ ${ }^{1}$ Science and Technology on Complex Electronic System Simulation Laboratory, Equipment academy, \\ Beijing, China \\ ${ }^{2}$ Jiuquan Satellite Launch Centre, Jiuquan, China
}

\begin{abstract}
Multi-Resolution Model and Simulation(MRMS) Technology is an important Aspect in the Complex large System Simulation field with important value to decrease model computation complexity to increase the simulation efficiency. Since the bottleneck of the MRMS at the aspect of Model consistence maintenance, aggregation- disaggregation(A/D) algorithm, chained disaggregation problem and so on, this paper proposed a non-consistence A/D MRMS method which won't seek overtop Model consistence and allows cross Resolution interaction at the accept range of caused errors. Introduced the basic idea and the Agent Model with the parameterization A/D algorithm and Battle Simulation experiment of Weapon Equipment SoS was took to validate it. The result indicated the ability of performance increase and the simulation errors caused by A/D operation can be controlled, What's more, this technology is easy to realize and so threw away a brick for the development of the MRMS application.
\end{abstract}

Keywords: MRMS, aggregation and disaggregation, Complex System, Weapon SoS, Battle Simulation.

\section{Introduction}

Since the development of the weapon equipment with high and new technology and the theory of information warfare, System-of-systems(SoS) confrontation gradually becomes the future's main battle pattern and battle simulation of SoS gradually becomes the main study aspect in the military field as a result. Compared with the traditional simulation system, SoS simulation system needs much more computation resource expense and possess larger computation complexity since the scale of SoS is larger, the included entities is more and the interaction relations within the entities are more complex which have restricted the development and application of the battle simulation technology for SoS to a great extent [1]. At present, raising a simulation technology suit to the large scale SoS simulation which can decrease the complexity of the simulation system and increase the performance efficiency to support the battle simulation of the SoS is a urgent need.

Resolution is the object's minimum distinguish scale and in the battle simulation field, it is mainly means the extent and amount of the model to depict the real world's detail. Multi-resolution model(MRM) is to build several resolution models at different resolution scales for the studied object and simulation which utilize the MRM technology is called Multi-Resolution simulation(MRS) and we can name them as Multiresolution Model and Simulation(MRMS). Now, theory of MRMS is said to be the most effective technology to deal with the complex system simulation questions and so the SoS as a complex large system becomes its main study target. However, studies about MRMS mainly concentrate on the basic theory aspect whereas the related applications are very few confined with the complex realize process and the problem of consistency maintenance. For that reason, this paper researched the battle simulation theory for SoS based on MRMS faced to the actual application and mainly studied a series of questions related with the actual application

+ Corresponding author. Tel.: 13081828682 .

E-mail address: anxf07@ sina.com. 
such as the A/D idea, A/D model and A/D algorithm, as a result, provided an effective and validated simulation study method for the large scale SoS based on MRMS.

\section{MRMS Technology}

\subsection{Basic idea}

Resolution selection is an unavoidable problem for all the model and simulation studies and so long as the resolution is smaller, the simulation result is more dependable. However, it is not the smaller the better since the model with too small resolution meets with higher computation resource expense for what the MRM framework comes into being. The main purpose of MRM is not only to decrease the computation complexity as low as possible [2] but also to maintain a perfect depiction of the object detail [3]. The basic idea of MRMS is implementing dynamic A/D to the entities accords to the simulation requirement since the Aggregation operation can diminish the scale of the entities of the simulation circle and the disaggregation operation can realize the correction of high and low resolution model with each other, at last decrease the simulation dispatch expense and at the same time doesn't affect the simulation realness too much. What's more, some authors [4] thought that the MRM technology is one of the important study direction of the simulation in the future.

\subsection{Related study and the main problems}

MRM technology is a hotspot in the simulation field and is also an important content. But the study for it is very hard since many viewpoints about it haven't reached agreement and many concepts have little conduct value. According to the related study, the standard method is the children model substitution technique, the A/D technique and the multiple expression technique all of which have their adaptive application scope. The children model substitution technique based on regression and fitting belongs to the static MRM technique without the dynamic A/D process. The multiple expression technique normally considers the existent multiple level character of the model itself or the objective multi-resolution depiction requirement and always needs much more computation resource expense. The A/D is a dynamic MRM technique since the model's resolution is varying all the time whose purpose is to decrease the simulation complexity and is used more widely.

So far, the A/D technology has gained a primary application at several fields. Paper [5] used the A/D technology to detect the train's security through simulation and the data interaction within the different resolutions was based on Pseudo A/D method which is not a real simulation entity. Paper [6] proposed a formalization description method of the DEVS simulation framework based on Multi-resolution technology and the A/D process of the multi-resolution entities, the consistence mapping function and the ensemble emergence phenomenon owing to the coupling of the children models were studied and the inter-operation matter was coped with especially. Start from the model's objective multi-resolution depiction requirement, Paper [7] mainly studied the A/D theory and the consistence maintenance problem through the middle model which owns the function of mapping and interaction. The above studies has important promotion significance to the MRM technology, however, these studies emphasis the model consistence too excessively and the middle mapping list must be built or the multiple resolutions of one entity must be run, for what reasons the purpose of raising the efficiency of the simulation system run at the opposite direction.

The USA national science commission thinks that the MRM is one of the most basic challenges of the modern modeling and simulation technology [8]. Now, the application of MRMS technology is restricted by a lot of problems such as the Aggregation mechanism, the Disaggregation mechanism and especially the consistence maintenance problem. What the consistence means is that when the different resolution entities want to interact, they must satisfied with the same resolution requirement through the process of Aggregation or Disaggregation. However, the interaction within different entities is very frequent and is not controllable and precognition in the actual simulation process for which reasons the A/D operation will be frequent extremely so that the computation resource is increased on the contrary. In fact, the transformation process of the different resolution entities is also a correction process with each other and so the deviation due to the Non-consistence interaction. As a result, a non-consistence A/D MRMS method which won't seek overtop 
Model consistence and allows cross Resolution interactions was proposed which is the main idea and innovation spot of this paper.

\section{Model Establish}

\subsection{Basic idea}

When the number of the simulated entities is very small, the advantage of the A/D technology cannot play a evident role where as the number of the simulated entities is very large (thousand, for example), the performance increase of it will be obvious. Thinking about the difficulty of consistence maintenance and other disadvantages aroused from it [6], the idea of this paper is that allowing the interaction between different resolutions in the error's allowable range and don't seeking overtop individual entities' consistence to avoid the extra computation resource expense in order to decrease the simulation computation complexity as far as possible.

That is, the simulation management of different resolutions is all the same and cross Resolution interactions are allowed which means the model management is independent with its resolution and the A/D operation is also independent with the model management but by the fixed $\mathrm{A} / \mathrm{D}$ circle $\mathrm{T}$. Although the simulation realness may be decreased due to the non-consistence interaction, the lost realness will be covered at some extent through the repeated dynamic $A / D$ process since the $A / D$ operation of different resolution entity is also a correction process with each other from the whole angle of view. Besides, the benefits of it will be a lot, for example, it needn't to keep the middle mapping list for different resolutions or keep a special resolution identification, therefore, it not only leave out the trouble of consistence maintenance but also avoid extra computation expense and so on. At the concrete process, the A/D operation is according to a cycle $T$ which is the simulation tick interval and a percentage $\lambda$ which is the percentage of the aggregation entities to the all equivalence entities. The Non-consistence A/D framework with two levels is as Fig. 1 showed, it can be known from it that the Disaggregation operation interval is $T / 2$ and the low resolution Agent is inherited from the high resolution Agent.

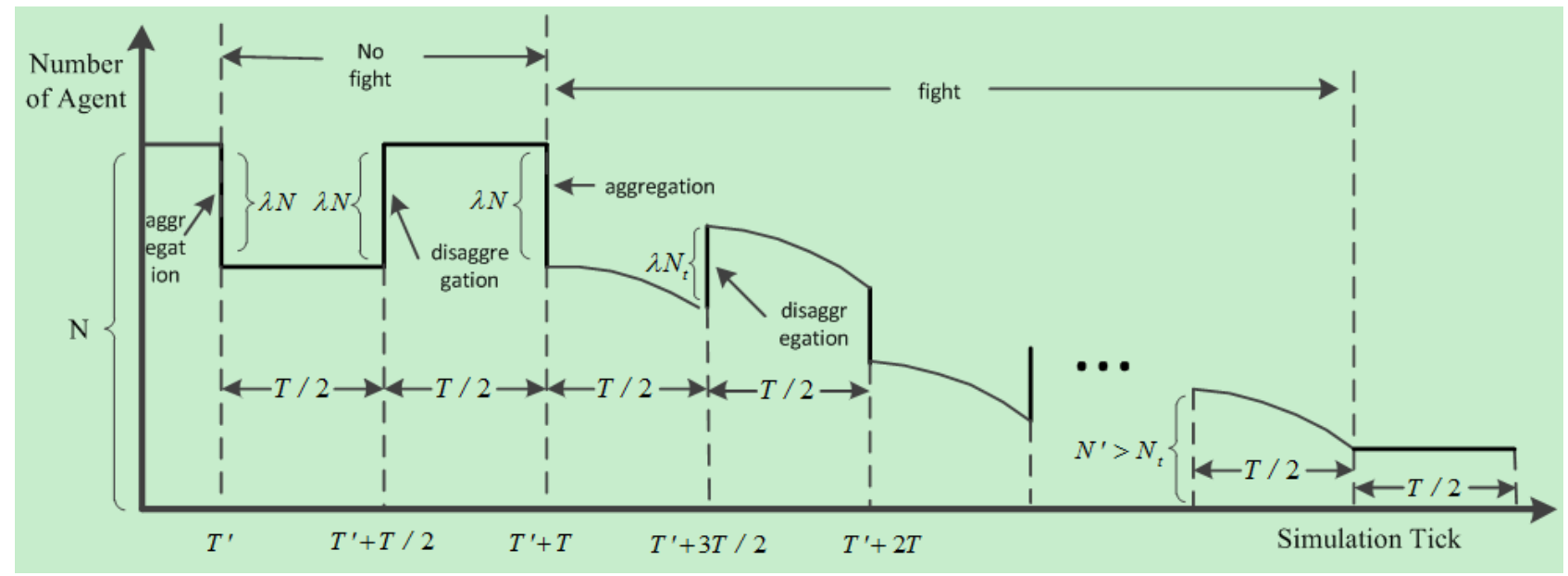

Fig. 1: Mechanism sketch of the Non-consistence A/D method.

\subsection{Basic Agent model oriented to SoS simulation}

Normally, Weapon equipment SoS includes the communication class, the scout class, the attack class, the supply class, the command class and the repair class, so in order to take a simulation study of the SoS, all these classes are included as follow introduction.

(1) Communication Agent

The function of Communication Agent is ensuring the message's transportation corresponding to the communication class in the Weapon Equipment SoS. Three kinds of communication Agent are designed which are ground communication Agent, air communication Agent and the sea communication Agent. The main performance parameter of it is communication range and the other classes must be in the communication range of anyone communication Agent if they want to send or receive messages. 
(2) Scout Agent

The function of Scout Agent is scouting about the battlefield and sending information to other Agents if there are enemy corresponding to the scout class in the Weapon equipment SoS. Three kinds of Scout Agent are designed which are ground Scout Agent, air Scout Agent and the sea Scout Agent. The main performance parameters of it are scout distance and scout probability.

(3) Attack Agent

The function of Attack Agent is attack corresponding to the attack class in the Weapon equipment SoS. Three kinds of attack Agent are designed which are ground attack Agent, air attack Agent and the sea attack Agent. The main performance parameters of it are attack distance, attack probability, attack range(all the Agents in the attack range will be attacked), attack ability(the number of health decrease when Agent is attacked by it), carrying ammunition amount and the biggest carrying ammunition amount.

(4) Supply Agent

The function of Supply Agent is Supplying ammunition to the attack Agent corresponding to the supply class in the Weapon equipment SoS. Three kinds of Supply Agent are designed which are ground Supply Agent, air Supply Agent and the sea Supply Agent. The main performance parameters of it are Supply distance and Supply ability (the supplying ammunition at one time).

(5) Repair Agent

The function of Repair Agent is recovering other Agent's health corresponding to the Repair class in the Weapon equipment SoS. Only one kind of Repair Agent is designed which is ground Repair Agent but it can repair all the other kind Agents. The main performance parameters of it are Repair distance and Repair rate (the health recovered at one time).

(6) Command Agent

The function of Command Agent is commanding other Agent's action according to decision policy corresponding to the Command class in the Weapon equipment SoS. Three kinds of Command Agent are designed which are ground Command Agent, air Command Agent and the sea Command Agent. Every formation has one command Agent and the subordinated Agent must follow the command Agent's action.

\subsection{Parameterization A/D algorithm}

Parameterization Dynamic A/D technology is in the common use, at the base of further developing it, this paper carried out the experiment validation. In fact, the A/D operation to Agent must coincide with several conditions such as the same class requirement, the same formation requirement, the same group requirement and the same resolution requirement. Besides, the most important principle is decreasing the information lost as little as possible. Then the Parameterization A/D algorithm for the scout Agent, the communication Agent, the attack Agent and the supply Agent is introduced as followed(since the repair Agent is normally less, the A/D operation for it is useless).

(1) Parameterization A/D algorithm for Scout Agent

The aggregated Agent named as low resolution Agent is inherited from the corresponding high resolution Agent at the concrete realize process through which to ensure the same attribute and function of the aggregated Agent with the high resolution Agent. The basic Parameterization A/D algorithm for location, health, velocity are all the same with other kinds Agent what the $A_{L}=\left(\sum_{i=1}^{N} a_{i L}\right) / \mathrm{N}, a_{x}=A_{x}+i \times d, a_{y}=A_{y}$, $a_{z}=A_{z}+j \times d, A_{H}=\delta \times \sum_{i=1}^{N} a_{i H}, a_{i H}=A_{H} / N, A_{V}=\sum_{i=1}^{N} a_{i V} / \mathrm{N}, a_{V}=A_{V}$ What $N$ is the number of the Aggregated Agents, ${ }^{a_{x}}$ is the value of axis ${ }^{x}$ of Agent's coordinate, $a_{y}$ is the value of axis $y$ of Agent's coordinate, $a_{z}$ is the value of axis $z$ of Agent's coordinate, $i=j=[-N / 2,-N / 2+1,-N / 2+2, \ldots, N / 2], d$ is the fixed distance, the coordinate generation process of Disaggregated Agent is similar with paper [9]. The other Parameterization A/D algorithms for Scout Agent is showed as Table I, What $\delta$ is the gain factor, The meaning of the same symbols are all the same in this paper.

Table I. Parameterization A/D Algorithms For Scout Agent

\begin{tabular}{ccccc}
\hline $\begin{array}{c}\text { Parameter } \\
\text { name }\end{array}$ & low resolution symbol & high resolution symbol & $\begin{array}{c}\text { Aggregation } \\
\text { algorithm }\end{array}$ & $\begin{array}{c}\text { Disaggregation } \\
\text { algorithm }\end{array}$ \\
\hline Scout distance & $A_{S}$ & $a_{S}$ & $A_{S}=\left(\sum_{i=1}^{N} a_{i s}\right) / N$ & $a_{S}=A_{S}$ \\
\hline
\end{tabular}


(2) Parameterization A/D algorithm for Communication Agent

It should be known that all the A/D algorithm of basic parameters included location, health, velocity and so on are same except the exclusive parameters. For the communication Agent, the only exclusive parameter is communicate range $A_{C}$ whose aggregation algorithm is $A_{C}=\delta \sum_{i=1}^{N} a_{i c}$ and disaggregation algorithm is $a_{i C}=A_{C} / N$.

(3) Parameterization A/D algorithm for attack Agent

The exclusive parameters of attack Agent are attack range ar, attack distance $a l$, attack ability $a k$, carrying ammunition $a a$ and the largest carrying ammunition $a m$. Their A/D algorithms are showed as Table II.

Table II. Parameterization A/D Algorithms for Attack Agent

\begin{tabular}{ccccc}
\hline Parameter name & $\begin{array}{c}\text { low resolution } \\
\text { symbol }\end{array}$ & high resolution symbol & Aggregation algorithm & $\begin{array}{c}\text { Disaggregation } \\
\text { algorithm }\end{array}$ \\
\hline Attack range & $A_{a r}$ & $a_{a r}$ & $A_{a r}=\sum_{i=1}^{N} a_{i a r} / N$ & $a_{i a r}=A_{a r}$ \\
Attack distance & $A_{a l}$ & $a_{a l}$ & $A_{a l}=\sum_{i=1}^{N} a_{i a l} / \mathrm{N}$ & $a_{i a l}=A_{a l}$ \\
Attack ability & $A_{a k}$ & $a_{a k}$ & $A_{a k}=\delta \sum_{i=1}^{N} a_{i a k}$ & $a_{i a k}=A_{a k} / \mathrm{N}$ \\
carrying ammunition & $A_{a a}$ & $a_{a a}$ & $A_{a a}=\delta \sum_{i=1}^{N} a_{i a a}$ & $a_{i a a}=A_{a a} / \mathrm{N}$ \\
Largest carrying & $A_{a m}$ & $a_{a m}$ & $A_{a m}=\sum_{i=1}^{N} a_{i a m}$ & $a_{i a m}=A_{a m} / \mathrm{N}$ \\
ammunition & & & & \\
\hline
\end{tabular}

(4) Parameterization A/D algorithm for Supply Agent

The exclusive parameters of Supply Agent are Supply distance $s l$, loading capacity $s v$, Supply speed $s r$, Their A/D algorithms are showed as Table III.

Table III. Parameterization A/D Algorithm for Supply Agent

\begin{tabular}{ccccc}
\hline Parameter name & low resolution symbol & high resolution symbol & Aggregation algorithm & $\begin{array}{c}\text { Disaggregation } \\
\text { algorithm }\end{array}$ \\
\hline Supply speed & $A_{s r}$ & $a_{s r}$ & $A_{s r}=\delta \sum_{i=1}^{N} a_{i s r}$ & $a_{i s r}=A_{s r} / N$ \\
Supply distance & $A_{s l}$ & $a_{s l}$ & $A_{s l}=\sum_{i=1}^{N} a_{i s l} / N$ & $a_{i s l}=A_{s l}$ \\
loading capacity & $A_{s v}$ & $a_{s v}$ & $A_{s v}=\delta \sum_{i=1}^{N} a_{i s v}$ & $a_{i s v}=A_{s v} / \mathrm{N}$ \\
\hline
\end{tabular}

\section{Simulation Validation}

To validate the Non-consistence A/D idea proposed through battle simulation of SoS based on red-blue confrontation, a distributed simulation platform for large scale SoS based on Agent and Java3D technology was developed [10]. To fully bring this dynamic A/D technology into play, the simulated entities is as many as possible.

\subsection{Experiment arrange}

The purpose of the experiment is to validate the Non-consistence A/D method's advantage at the efficiency increasing for the large scale system's simulation. The basic idea is comparing the time consumed every simulation tick at different conditions which is taking A/D and not taking A/D. The combat scenario is red-blue confrontation and the architecture of the red SoS is showed as Table IV and the blue group is the same as the red. It is known from the table that there are 902 Agents need to be managing at all and the tremendous computation complexity without dynamic A/D is imaginable. 
Table IV. Architecture Set up of the Red Group SoS

\begin{tabular}{ccccc}
\hline Order number & Agent class & Space class & team & size \\
\hline 1 & scout & ground & 1 & 100 \\
2 & attack & ground & 1 & 200 \\
3 & communicate & ground & 1 & 150 \\
4 & supply & ground & 1 & 50 \\
5 & repair & ground & 1 & 1 \\
\hline
\end{tabular}

\subsection{Result analysis}

The experiment is carried out more than one time to improve the result's reliability. The Aggregation decrease rate $\delta$ is fixed to 0.5 and the $\mathrm{A} / \mathrm{D}$ circle $\mathrm{T}$ with the $\mathrm{A} / \mathrm{D}$ scale $\lambda$ is especially studied through changing the value of them separately. Besides, the max simulation tick is fixed to 100 to prevent the simulation is finished too early. Fig. 2 is the number changing of the Agent with the simulation tick at different A/D scale and the lateral axis is simulation tick and the vertical axis is the number of the Agent. The tick of carrying A/D is known from the figure obviously and the bigger is the $\lambda$, the changing extent of the Agent number is bigger, besides, the interval between two Aggregation time point is just a A/D circle T. besides, along with the simulation in progress, the A/D scale is decreasing since the Agent is diminish by fighting and when the number of Agent is less than the least $A / D$ requirement, the $A / D$ operation will stop too since the computation resource expense is more than the efficiency increase by it.

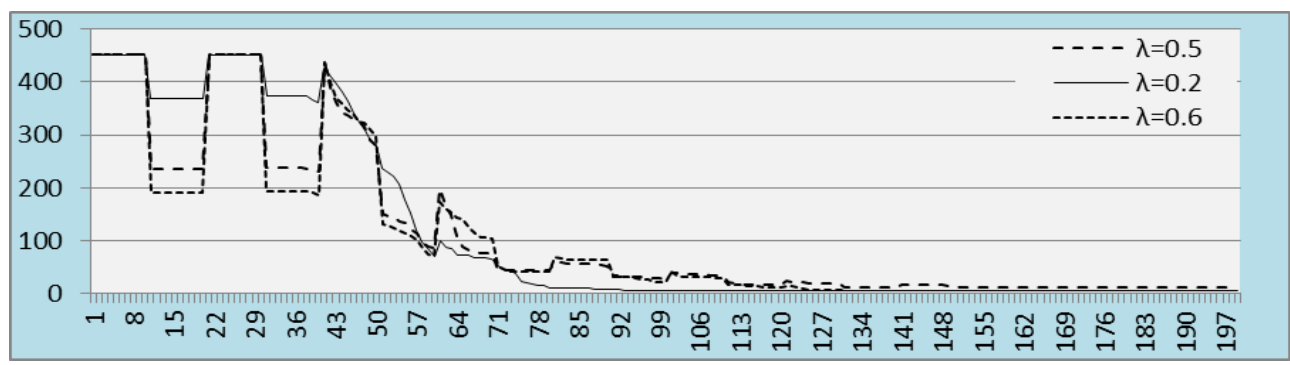

Fig. 2: The number of the Agent with the simulation tick at different A/D scale.

The average time $\bar{t}$ consumed at one simulation tick is $1.17 \mathrm{~s}$ without $\mathrm{A} / \mathrm{D}$ which is much less at the condition of $\mathrm{A} / \mathrm{D}$ operation corresponding to the experiment result at Table $\mathrm{V}$. It can be known from the result that the most important parameter affect the simulation efficiency is A/D scale whereas the A/D circle has nothing to do with it. When the A/D circle is fixed to 20, the simulation efficiency is increasing with the $\mathrm{A} / \mathrm{D}$ scale and when the scale is up to 0.9 , the enhanced efficiency compared to status without A/D nearly reaches to the limit as is $72.9 \%$. the reason is that the larger is the A/D scale, the larger is the decreased entities amount in the whole simulation period, as a result the efficiency is increased which indicates the validation of this method at the aspect of performance strengthen adequately.

Besides, we studied the simulation errors owing to A/D process since there must be information lose and this is also important to the model's validation. Here, we analyzed the simulation errors affect by the A/D scale $\lambda$ and the Aggregation decrease rate $\delta$. The structure of the two groups is same as the Table IV but only the red group took the A/D operation so the battle result affect by A/D operation can by reflected. What's more, to produce the victory and defeat as far as possible, max simulation tick is fixed to 1000 .

We used the victory possibility difference to show the simulation errors since the victory possibility of the red group should be the same as the blue group if their military set up is same. The victory possibility difference is calculated as Verror $=|r v-b v|$ where the $r v$ is victory possibility of the red group and the $b v$ is the victory possibility of the blue group. So the Verror is the smaller the better. The simulation result is showed as the Table VI, it can be known from the table that Verror $=0.066$ is the lowest when $\lambda$ is fixed to 0.3 and $\delta$ is 0.5 whereas the Verror is increased when the $\delta$ is higher or lower than 0.5 . When the $\delta$ is fixed to 0.5 , the Verror is the lowest when $\lambda$ is 0.3 whereas the Verror is increased when the $\lambda$ is higher or lower than 0.3. all 
in all, when the T is 20 and the $\lambda$ is 0.3 and the $\delta$ is 0.5 , the simulation errors caused by A/D operation is the lowest which indicated the validation of the Non-consistence A/D Technology method.

Table V.Experiment Result of the Battle Simulation with A/D Operation

\begin{tabular}{cccccc}
$\mathbf{T}$ & $\lambda$ & $\bar{t}(\mathbf{s} /$ tick $)$ & $\mathbf{T}$ & $\lambda$ & $\bar{t}(\mathbf{s} /$ tick $)$ \\
\hline 10 & 0.3 & $0.651 \mathrm{~s}$ & 20 & 0.1 & $1.031 \mathrm{~s}$ \\
20 & 0.3 & $0.75 \mathrm{~s}$ & 20 & 0.3 & $0.74 \mathrm{~s}$ \\
30 & 0.3 & $0.71 \mathrm{~s}$ & 20 & 0.5 & $0.54 \mathrm{~s}$ \\
40 & 0.3 & $0.772 \mathrm{~s}$ & 20 & 0.7 & $0.383 \mathrm{~s}$ \\
50 & 0.3 & $0.757 \mathrm{~s}$ & 20 & 0.9 & $0.317 \mathrm{~s}$ \\
\hline
\end{tabular}

Table VII.Experiment Result of the Simulation Errors Caused By A/D Operation

\begin{tabular}{cccccc}
\hline $\mathrm{T}$ & $\lambda$ & $\delta$ & $r v$ & $b v$ & Verror \\
\hline 20 & 0.3 & 0.4 & $8 \%$ & $39 \%$ & 0.31 \\
20 & 0.3 & 0.5 & $16.67 \%$ & $23.33 \%$ & 0.066 \\
20 & 0.3 & 0.6 & $60 \%$ & $13.33 \%$ & 0.4667 \\
20 & 0.3 & 0.7 & $54.55 \%$ & $21.21 \%$ & 0.3334 \\
20 & 0.3 & 0.8 & $7.69 \%$ & $66.67 \%$ & 0.5898 \\
20 & 0.3 & 0.9 & $4 \%$ & $88 \%$ & 0.84 \\
20 & 0.2 & 0.5 & $50 \%$ & $26.7 \%$ & 0.233 \\
20 & 0.4 & 0.5 & $3 \%$ & $46.67 \%$ & 0.436 \\
20 & 0.5 & 0.5 & 0 & $38.89 \%$ & 0.3889 \\
20 & 0.6 & 0.5 & 0 & $50 \%$ & 0.5 \\
20 & 0.7 & 0.5 & 0 & $50 \%$ & 0.5 \\
\hline
\end{tabular}

\section{Conclusion}

Study of MRMS is an important task in the field of complex large system simulation since it is valuable to the decrease of the model's computation complexity and increase the simulation efficiency. However, the present study of MRMS whose purpose is to decrease the simulation complexity is still in the stage of theory and concept argument because of the complex realizes process and the difficult consistence maintenance operation. For that reason, this paper proposed a new non-consistence A/D MRMS method which won't seek overtop Model consistence and allows cross Resolution interaction at the accepted range of caused errors. The battle simulation for SoS indicated the validation of this method at the aspect of simulation performance increase and the simulation errors control which is meaningful to the engineering applications of MRMS.

\section{References}

[1] J. X. Huang, Q. Li, Q. Jia, et al. "Research on ABMS-based SoS effectiveness evaluation framework". Systems Engineering and Electronics, 2011,33(8):1794-1798.

[2] A. Meystel, M. Bathija, "Multi resolutional Planning: Using the Randomized Tessellation of the State Space". ISRA 2002.

[3] Z. Guan, C. Wang, J. Huang, et al. "Optimization of Manufacturing Systems using Genetic Search and MutiResolution Simulation”. 2010 8th IEEE International Conference on Control and Automation Xiamen, China, June 9-11, 2010. 
[4] L. BoHu, "Modeling and Simulation in China-Present and Future", Proceedings of 2002 Asian Simulation Conference. Nov.3-6,2002.Shanghai,China.

[5] X. G. Chen, G. C. Bai, "Multi-Resolution Entities Aggregation and Disaggregation Method for Train Control System Modeling and Simulation Based on HLA", 2014 IEEE 17th International Conference on Intelligent Transportation Systems (ITSC)October 8-11, 2014. Qingdao, China.

[6] Y. Li, B. H. Li, X. F. Hu ,et al. "Formalization of Multi-Resolution Modeling Based on Dynamic Structure DEVS", International Conference on Information Science and Technology March 26-28, 2011 Nanjing, Jiangsu, China.

[7] S. G. Wei, B. G. Cal, S. H. LI, et al, "Multi-resolution simulation strategy and its simulation implementation of Train Control System". IEEECONF,2011.

[8] Y. B. DU, J. J. YANG, W. LV, et al, "Study of Relevant Conceptions about Multi-resolution Modeling and Simualtion”,Journal of System Simulation, 2008,20(6):1386-1389.

[9] J. W. Li, "Research on Application of Multi-resolution Modeling in Carrier Combat Group Operation Simulation",Journal of System Simulation, 2013,25(8):1924-1929.

[10] X. F. Yan, X. M. Li, D. Liu, et al, "MR-OA: An effective framework for NCW study based on multi-Agent simulation", 2015 Fifth International Conference on Instrumentation \& Measurement, Computer, Communication and Control, China,Haerbin,2015:1961-1966. 